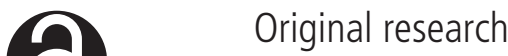

Randomised comparison of postpolypectomy

OPEN ACCESS

\section{surveillance intervals following a two-round baseline colonoscopy: the Japan Polyp Study Workgroup}

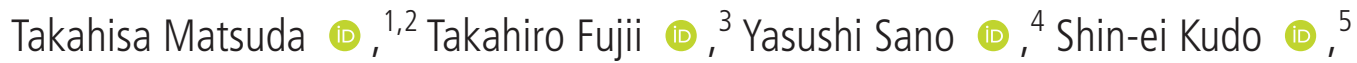 \\ Yasushi Oda, ${ }^{6}$ Kinichi Hotta (1) , ${ }^{7}$ Tadakazu Shimoda, ${ }^{8}$ Yutaka Saito (ㄷ) , \\ Nozomu Kobayashi, ${ }^{9}$ Masau Sekiguchi (1) , 1,2 Kazuo Konishi 두, ${ }^{10}$ \\ Hiroaki Ikematsu (1), ${ }^{11}$ Hiroyasu lishi (1), ${ }^{12}$ Yoji Takeuchi (1) ,12

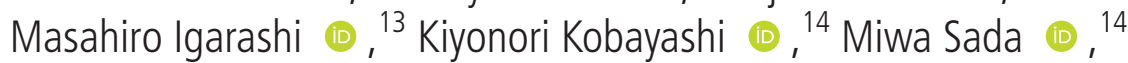 \\ Yuichiro Yamaguchi, ${ }^{7}$ Kiwamu Hasuda, ${ }^{15}$ Tomoaki Shinohara (1), ${ }^{16}$

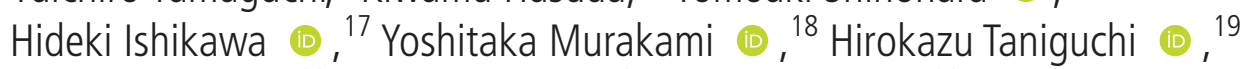 \\ Takahiro Fujimori, ${ }^{20}$ Yoichi Ajioka @i ${ }^{21}$ Shigeaki Yoshida ${ }^{22}$
}

For numbered affiliations see end of article.

\section{Correspondence to}

Dr Takahisa Matsuda, Cancer Screening Center, National

Cancer Center Hospital, Chuoku, Tokyo, Japan;

tamatsud@ncc.go.jp

Received 27 May 2020 Revised 13 September 2020

Accepted 20 September 2020 Published Online First

2 November 2020
Check for updates

(C) Author(s) (or their employer(s)) 2021. Re-use permitted under CC BY-NC. No commercial re-use. See rights and permissions. Published by BMJ.

\footnotetext{
To cite: Matsuda T, Fujii T, Sano Y, et al. Gut

2021:70:1469-1478.
}

\section{ABSTRACT}

Objective To assess whether follow-up colonoscopy after polypectomy at 3 years only, or at 1 and 3 years would effectively detect advanced neoplasia (AN), including nonpolypoid colorectal neoplasms (NP-CRNs).

Design A prospective multicentre randomised controlled trial was conducted in 11 Japanese institutions. The enrolled participants underwent a two-round baseline colonoscopy (interval: 1 year) to remove all neoplastic lesions. Subsequently, they were randomly assigned to undergo follow-up colonoscopy at 1 and 3 years (2-examination group) or at 3 years only (1-examination group). The incidence of AN, defined as lesions with low-grade dysplasia $\geq 10 \mathrm{~mm}$, high-grade dysplasia or invasive cancer, at follow-up colonoscopy was evaluated.

Results A total of 3926 patients were enrolled in this study. The mean age was 57.3 (range: 40-69) years, and $2440(62 \%)$ were male. Of these, 2166 patients were assigned to two groups (2-examination: 1087, 1-examination: 1079). Overall, we detected 29 AN in 28 patients at follow-up colonoscopy in both groups. On per-protocol analysis (701 in 2-examination vs 763 in 1-examination group), the incidence of AN was similar between the two groups ( $1.7 \%$ vs $2.1 \%, p=0.599$ ). The results of the non-inferiority test were significant $(p=0.017$ in per-protocol, $p=0.001$ in intention-to-treat analysis). NP-CRNs composed of dominantly of the detected AN $(62 \%, 18 / 29)$, and most of them were classified into laterally spreading tumour non-granular type $(83 \%, 15 / 18)$.

Conclusion After a two-round baseline colonoscopy, follow-up colonoscopy at 3 years detected AN, including NP-CRNs, as effectively as follow-up colonoscopies performed after 1 and 3 years.

\section{INTRODUCTION}

Colorectal cancer (CRC) is the third most common cancer in men and the second in women worldwide. ${ }^{1}$

\section{Significance of this study}

What is already known on this subject?

- The US National Polyp Study demonstrated that a singular follow-up colonoscopy at 3 years postpolypectomy is equally effective in detecting advanced neoplasia (AN) as are follow-up colonoscopies at 1 and 3 years after polypectomy.

- Despite the improvement of postpolypectomy surveillance programmes, postcolonoscopy colorectal cancer (PCCRC) remains problematic, giving rise to the need for postpolypectomy surveillance colonoscopy with a focus on easily overlooked lesions such as nonpolypoid colorectal neoplasms (NP-CRNs).

What are the new findings?

- The present study demonstrated the equivalent accuracy of detecting AN with a single surveillance colonoscopy at 3 years, compared with two surveillance colonoscopies at 1 and 3 years postpolypectomy, even considering NP-CRNs.

- Even after a two-round baseline colonoscopy, AN mainly composed of NP-CRNs, especially, laterally spreading tumour, non-granular type (LST-NG), were detected at follow-up colonoscopy. ANs were more frequently detected in individuals in whom LST-NG had been treated at baseline colonoscopy.

Colonoscopy is considered as the gold standard to prevent CRC since its efficacy in reducing the incidence and mortality of CRC by detection and removal of adenomatous polyps has been widely demonstrated. ${ }^{2-6}$ For colonoscopy to be efficiently utilised, it is essential to establish the optimal intervals for surveillance colonoscopy. The US National 
Significance of this study

How might it impact on clinical practice in the foreseeable future?

- It was confirmed that postpolypectomy surveillance colonoscopy intervals are not required to be shorter than 3 years, even with consideration of NP-CRNs, which may prevent the overuse of surveillance colonoscopy.

- The clinical importance of NP-CRNs, particularly LST-NG, was demonstrated in this study. Further studies are expected to clarify their relationship with PCCRC, including their potential role as precursors of PCCRC.

Polyp Study (NPS) is a monumental study to assess postpolypectomy surveillance colonoscopy intervals. This randomised controlled trial demonstrated that a singular follow-up colonoscopy at 3 years postpolypectomy is equally effective in detecting advanced colorectal neoplasia as follow-up colonoscopies at 1 and 3 years after polypectomy. ${ }^{7}$ Based on the NPS and other subsequent studies, ${ }^{8-10}$ the recommendations on postpolypectomy surveillance have reached their current form as described in the recent guidelines. ${ }^{11-13}$ Despite the improvement of postpolypectomy surveillance programmes, with considerations for not only adenomatous polyps but also serrated lesions, ${ }^{14}$ postcolonoscopy colorectal cancer (PCCRC) remains a problem. ${ }^{15-18}$ In this context, we hypothesised that nonpolypoid colorectal neoplasms (NP-CRNs), which have not been fully examined in previous studies, including the NPS, are the major cause of PCCRC. This is because these easily overlooked lesions are known to have a greater malignancy potential than polypoid lesions. ${ }^{19-22}$

In the present study, we assessed afresh the hypothesis investigating the NPS, with a special focus on NP-CRNs. The present study aimed to examine whether a single follow-up colonoscopy at 3 years will detect clinically important colorectal neoplastic lesions, including NP-CRNs, as effectively as two follow-up colonoscopies at 1 and 3 years. Uniquely, in this study, a two-round baseline colonoscopy was performed to ensure the removal of all neoplastic lesions, including NP-CRNs, before randomisation. This process enabled a more accurate comparison between the two surveillance intervals.

\section{METHODS}

\section{Study design and participants}

The Japan Polyp Study (JPS) was conducted as a prospective multicentre randomised controlled trial involving 11 Japanese referral institutions. Information regarding the protocol was provided to all participants, and informed consent was obtained from all participants before each intervention. A detailed description of the study rationale and design has been provided in previous publications and is available online. ${ }^{2324}$ Patients and the public were not involved in the design, analyses and interpretation of this study.

Between February 2003 and December 2006, individuals aged 40-69 years who were referred to participating institutions to undergo colonoscopy for any reason were enrolled in this study. Patients with a personal history of polypectomy with unknown histology, invasive CRC colectomy, familial adenomatous polyposis, Lynch syndrome or inflammatory bowel disease were excluded. After enrolment, participants underwent a two-round baseline colonoscopy (first colonoscopy and second colonoscopy; interval: 1 year). If invasive CRC, sessile adenoma $\geq 30 \mathrm{~mm}$ (which were excluded considering a higher risk of local recurrence after endoscopic piecemeal resection), or inflammatory bowel disease were detected at any of the two-round baseline colonoscopies, the affected patients were excluded from this study at that time. After the two-round baseline colonoscopy, the patients were randomly assigned to undergo follow-up colonoscopies at 1 and 3 years (2-examination group) or at 3 years only (1-examination group) to compare the incidence of advanced neoplasia (AN). The AN was defined as any lesion with lowgrade dysplasia (LGD) $\geq 10 \mathrm{~mm}$, high-grade dysplasia (HGD) or invasive cancer. ${ }^{12} 13$ The JPS Workgroup excluded lesions with villous components from $\mathrm{AN}$, as did in the UK guidelines. This is because the pathological evaluation of villous lesions could be influenced by the pathologist's interpretation and might not be completely objective. ${ }^{12132526}$

\section{Scheduled examination period and handling of patients}

Follow-up colonoscopies were planned for a period of 3-6 months before and after the designated dates, which were 1 and 3 years (in 2-examination group) and 3 years only (in 1-examination group) after randomisation. A per-protocol analysis was assessed strictly on the basis of the data of patients who underwent follow-up colonoscopies during this period. Patients who received follow-up colonoscopies beyond this period were not interpreted to be dropouts in an intention-to-treat analysis.

\section{Sample size calculation and statistical analysis}

To determine the sample size required for a non-inferiority trial, the incidence of $\mathrm{AN}$ was set at 3\% at surveillance colonoscopy of 3 years after a two-round baseline colonoscopy, based on the results of the NPS. The non-inferiority margin was set at $2 \%$, the one-sided alpha at $2.5 \%$, and the statistical power at $80 \%$. For each group, 1142 patients were required. Thus, a total of 2284 patients were required for the study. Statistical analyses were performed using SAS V.9.4 (SAS). Data were presented as mean, standard deviation (SD) or frequency (\%). Univariate logistic regression was used to estimate the crude ORs and their 95\% CIs, which showed the relation between the risk factors and AN. All $p$ values were two-tailed, and values of $p<0.05$ were considered statistically significant.

\section{Randomisation}

Dynamic allocation using the minimisation method was used for the randomisation in this study. Five allocation factors were employed as follows: (1) institution, (2) gender (male/ female), (3) age group ( $<60$ years/ $\geq 60$ years), (4) CRC risk based on the findings of a two-round baseline colonoscopy and (5) history of colonoscopy (present/ absent).

\section{Colonoscopy, pathological diagnosis and data collection}

All procedures were performed by specialised endoscopists who had performed more than 500 colonoscopies before the study period. High-definition video endoscopes were used for all examinations, and chromoendoscopy and magnification were utilised for endoscopic diagnosis. Bowel preparation was conducted using $1.8 \mathrm{~L}$ of magnesium citrate (isotonic solution) or $2 \mathrm{~L}$ of polyethylene glycol at the hospital on the day of the colonoscopy. If necessary, additional magnesium citrate or polyethylene glycol was provided. The quality of the bowel cleansing was graded using a modified Aronchick scale, as follows: excellent (>95\% of mucosa seen), good (clear liquid covering up to $25 \%$ of the mucosa, but $>90 \%$ of mucosa seen), fair (semisolid stool could not be suctioned, but $>90 \%$ of mucosa seen) or poor 
(repeat preparation needed). ${ }^{27}$ Patients were sedated with midazolam, diazepam or additional pethidine hydrochloride when requested by the patients. For the patients without contraindications, scopolamine butylbromide or glucagon was administered to suppress bowel peristalsis.

All detected lesions were documented by the location, size, and macroscopic type, according to the Paris classification. ${ }^{28}$ Moreover, all flat elevated lesions $\geq 10 \mathrm{~mm}$, except serrated lesions, were classified as either laterally spreading tumour, non-granular type (LST-NG) or granular type (LST-G). ${ }^{29-31}$ The lesion size was calibrated by comparisons with the closed cups of a biopsy forceps (approximately $2.5 \mathrm{~mm}$ ). All detected lesions diagnosed as neoplastic lesions and right-sided large serrated lesions $\geq 10 \mathrm{~mm}$ were removed endoscopically. The fixed specimens were subjected to histological examination. The reference standard was histopathology using standard H\&E staining. An independent pathology review team was introduced to re-examine all AN retrospectively, and for each lesion, a consensus among the four pathology reviewers (TShim, HT, TFujim and YA) was reached. The histopathological and endoscopic classifications adopted for this study were based on the WHO classification $^{32}$ and the Japanese Society for Cancer of the Colon and Rectum system, ${ }^{33}$ respectively. All data obtained, on the basis of the study protocol, were overseen by Medical Research Support (Osaka, Japan).

\section{RESULTS}

\section{Study population}

Of 4752 referrals, 3926 patients with a mean age of 57.3 years (range; 40-69), among whom 2440 (62\%) were male, were enrolled in this study. Of these, 2757 patients who underwent a two-round baseline colonoscopy (first colonoscopy and second colonoscopy) with the removal of all neoplastic lesions and rightsided large serrated lesions, were eligible. Among the eligible patients, 2166 were assigned to a randomised group (patients with neoplasia) and 591 patients to a non-randomised group (patients without neoplasia) as shown in figure 1. During the randomisation process, 1087 patients were assigned to undergo follow-up colonoscopies at 1 and 3 years postpolypectomy (2-examination group), whereas 1079 patients were assigned to undergo a singular follow-up colonoscopy at 3 years postpolypectomy (1-examination group). Of these, 169 patients in the 2-examination group and 142 patients in the 1-examination group underwent follow-up colonoscopy beyond the designated period. In the 2-examination and 1-examination groups, 217 and 174 patients, respectively, were lost to follow-up. In total, 1464 patients (male: 958, female: 506; 701 in the 2-examination group and 763 in the 1-examination group) were evaluated in the per-protocol analysis. The characteristics of eligible patients at randomisation are shown in table 1 . Patients' baseline characteristics and findings of their two-round baseline colonoscopies were similar between the two groups.

\section{Overall findings at follow-up colonoscopy after randomisation}

During the follow-up colonoscopy after randomisation, 351 $(50.1 \%)$ patients in the 2-examination group and $289(37.9 \%)$ patients in the 1-examination group had neoplastic lesions, $(\mathrm{p}<0.001)$. Of these, $12(1.7 \%)$ patients in the 2-examination group and $16(2.1 \%)$ patients in 1-examination group had AN in the per-protocol analysis $(p=0.599)$ as shown in table 2 . On the basis of this result, the non-inferiority of the 1-examination group compared with the 2-examination group was demonstrated $_{2}$ as $\mathrm{p}=0.017$ in the per-protocol analysis, and $\mathrm{p}=0.001$ in the intention-to-treat analysis (figure 2).

\section{Clinicopathological characteristics of metachronous AN}

In total, $29 \mathrm{AN}$ in 28 patients (male: 20 (2.1\%), female: 8 (1.6\%), mean age: 60.6 years old) were detected during a 3 -year follow-up period in both groups. Among all AN, there were 6 LGD $\geq 10 \mathrm{~mm}, 5 \mathrm{HGD}$ and 1 invasive cancer (online supplemental figure 1) in 12 patients in the 2-examination group. In the 1-examination group, there were 9 LGD $\geq 10 \mathrm{~mm}$ and $8 \mathrm{HGD}$ in 16 patients. In the 2-examinationm group, $6 \mathrm{AN}$ were detected in the first and second follow-up colonoscopies,

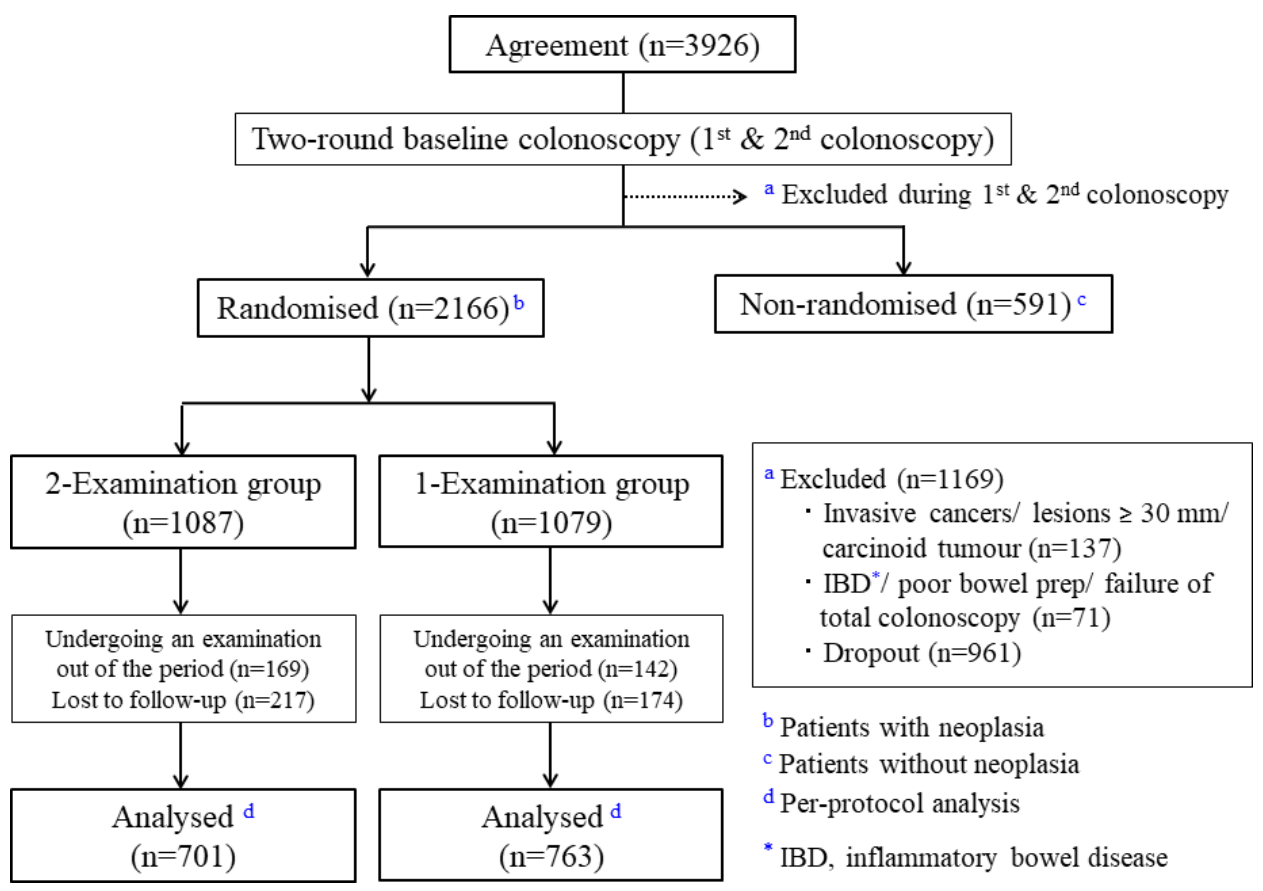

Figure 1 Study flow diagram. 
Table 1 Characteristics of the patients at randomisation

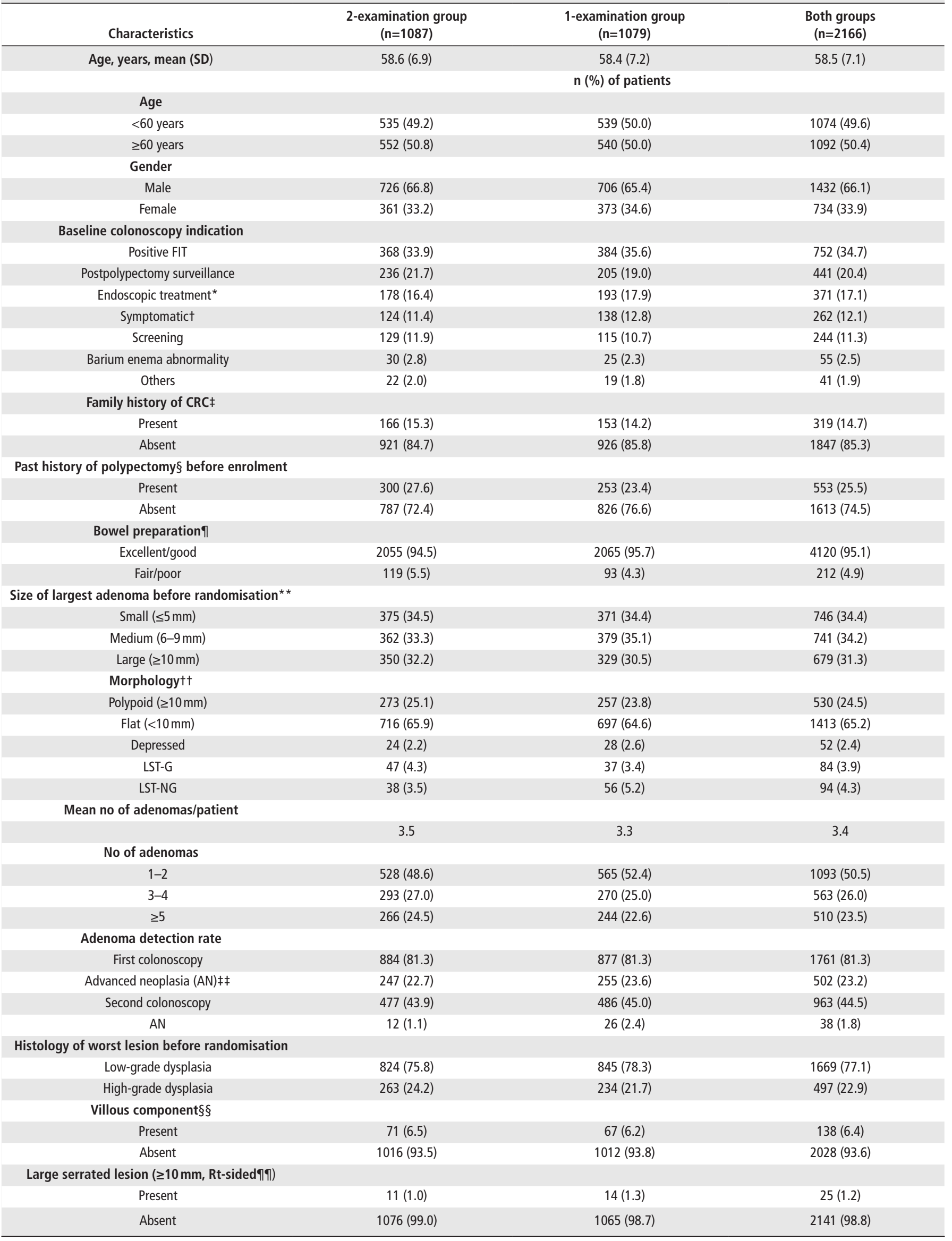


Table 1 Continued

\begin{tabular}{ccc}
\hline Characteristics & $\begin{array}{c}\text { 2-examination group } \\
(\mathrm{n}=1087)\end{array}$ & $\begin{array}{c}\text { 1-examination group } \\
(\mathrm{n}=1079)\end{array}$ \\
\hline $\begin{array}{c}\text { No of examinations after enrolment until colon } \\
\text { was polyp-free }\end{array}$ & & \\
2 & $1050(96.6)$ & $1038(96.2)$ \\
$\geq 3$ & $37(3.4)$ & $41(3.8)$ \\
\hline
\end{tabular}

*Referred from other hospitals for endoscopic treatment.

†Abdominal pain, constipation and bloody stool, among others.

¥First degree relative with CRC.

$\S$ Past history of polypectomy for neoplastic lesions before enrolment.

ๆData from all first/ second colonoscopy.

**Data from past history of colonoscopy and first/ second colonoscopy.

††Polypoid; 0-Ip, 0-Isp, 0-Is type, Flat; 0-Ila type, Depressed; 0-Ila+Ilc, 0-Ilc type.

$\ddagger \ddagger$ Any lesion with low-grade dysplasia $\geq 10 \mathrm{~mm}$, high-grade dysplasia.

$\S \S$ Tubulovillous adenoma and villous tumour.

१११Caecum, ascending colon, transverse colon.

AN, advanced neoplasia; CRC, colorectal cancer ; FIT, fecal immunochemical test; LST-G, laterally spreading tumour, granular type; LST-NG, laterally spreading tumour, nongranular type.

Table 2 Findings at follow-up colonoscopy after randomisation (per-protocol analysis)

\begin{tabular}{|c|c|c|c|c|}
\hline \multirow{3}{*}{ Finding } & \multicolumn{2}{|c|}{ 2-examination group } & \multirow{3}{*}{$\begin{array}{c}\text { 1-examination group } \\
\text { Follow-up examination } \\
(n=763) \\
\end{array}$} & \multirow{3}{*}{$P$ value } \\
\hline & First follow-up examination & Second follow-up examination & & \\
\hline & \multicolumn{2}{|c|}{$(\mathrm{n}=701)$} & & \\
\hline Polyps & $295(42.1)$ & $246(35.1)$ & $331(43.4)$ & $<0.001$ \\
\hline Any neoplasia detected & $244(34.8)$ & $218(31.1)$ & $289(37.9)$ & $<0.001$ \\
\hline \multicolumn{5}{|c|}{$351(50.1)$} \\
\hline Small $(\leq 5 \mathrm{~mm})$ & $205(29.2)$ & $168(24.0)$ & $208(27.3)$ & $<0.001$ \\
\hline Medium (6-9 mm) & $34(4.9)$ & $44(6.3)$ & $67(8.8)$ & 0.135 \\
\hline Large ( $\geq 10 \mathrm{~mm})$ & $5(0.7)$ & $6(0.9)$ & $14(1.8)$ & 0.703 \\
\hline \multicolumn{5}{|l|}{ Morphology* } \\
\hline Polypoid ( $\geq 10 \mathrm{~mm}$ ) & $2(0.3)$ & $2(0.3)$ & $3(0.4)$ & 0.646 \\
\hline \multicolumn{5}{|l|}{ Villous component $\dagger$} \\
\hline Present & $0(0)$ & $0(0)$ & $1(0.1)$ & 1.000 \\
\hline \multicolumn{5}{|c|}{ Large serrated lesion ( $\geq 10 \mathrm{~mm}$, Rt-sided $\ddagger$ ) } \\
\hline Present & $3(0.4)$ & $4(0.6)$ & $12(1.6)$ & 0.345 \\
\hline \multicolumn{5}{|l|}{ Dysplasia and invasive cancer§ } \\
\hline Low-grade dysplasia & $240(34.2)$ & $215(30.7)$ & $279(36.6)$ & $<0.001$ \\
\hline High-grade dysplasia & $3(0.4)$ & $2(0.3)$ & $8(1.0)$ & 0.514 \\
\hline Invasive cancer & $0(0)$ & $1(0.1)$ ๆ & $0(0)$ & 0.958 \\
\hline Others & $1(0.1)^{* *}$ & $0(0)$ & $2(0.3)+t$ & 1.000 \\
\hline \multicolumn{5}{|l|}{ Advanced neoplasiał‡ } \\
\hline No of lesions & 6 & 6 & 17 & 0.599 \\
\hline
\end{tabular}

*Polypoid; 0-Ip, 0-Isp, 0-Is type, Flat; 0-Ila type, Depressed; 0-Ila+Ilc, 0-Ilc type.

tTubulovillous adenoma.

$\ddagger$ Caecum, ascending colon, transverse colon.

$\S$ Histology of worst lesion.

१T2NOMO (Duke's stage B1) (online supplemental figure 1).

**1 Carcinoid tumour.

t†1 Carcinoid tumour, 1 Malignant lymphoma.

$\ddagger \ddagger$ Any lesion with low-grade dysplasia $\geq 10 \mathrm{~mm}$, high-grade dysplasia or invasive cancer.

LST-G, laterally spreading tumour, granular type; LST-NG, laterally spreading tumour, non-granular type. 


\section{2-examination better}

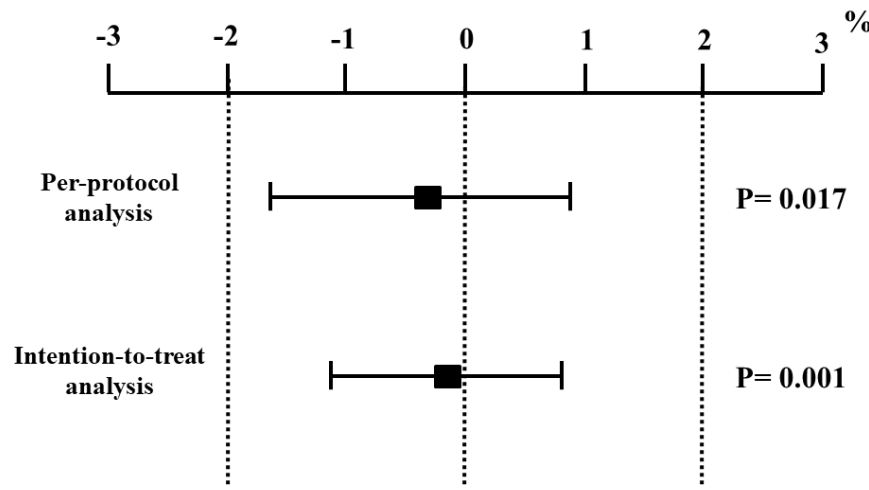

Figure 2 Differences in the incidence.

respectively. Concerning location, 13 (45\%) AN were located in the right-sided colon, and $16(55 \%)$ AN were observed in the left-sided colon. Morphologically, 18 (62\%) AN were classified as NP-CRN, $15(83 \%)$ of which were LST-NG lesions (online supplemental figure 2). No significant differences in the clinicopathological characteristics of AN were observed between the two groups (table 3 ).

\section{Risk factors associated with metachronous AN}

In the univariate analysis, the number of adenomas $(\geq 5)$ before randomisation (OR 2.84, 95\% CI 1.37 to 5.91 ), the presence of flat lesions $<10 \mathrm{~mm}$ (OR 3.04, 95\% CI 1.01 to 12.11) and LST-NG lesions (OR 6.61, 95\% CI 2.11 to 17.61$)$ treated at baseline colonoscopies, and family history of CRC (OR 2.39, 95\% CI 1.07 to 5.35 ) were associated with an increased risk of AN. No other clinicopathological factors were statistically significant as risk factors for AN (table 4).

\section{DISCUSSION}

To our knowledge, this is the first randomised controlled trial assessing the incidence of AN after a two-round baseline colonoscopy with removal of all neoplastic lesions. The present study demonstrated the equivalent accuracy of detecting clinically important neoplastic lesions with a single surveillance colonoscopy at 3 years, and two surveillance colonoscopies at 1 and 3 years postpolypectomy. The finding indicates that it is

Table 3 Clinicopathological characteristics of metachronous advanced neoplasia

\begin{tabular}{|c|c|c|c|c|c|c|}
\hline Group/ examination & Age & Gender & Location* & Morphology $\dagger$ & Size $(\mathrm{mm})$ & Histology \\
\hline \multirow{6}{*}{$\begin{array}{l}\text { 2-examination/ } \\
\text { first F/U }\end{array}$} & $60 \mathrm{~s}$ & $\mathrm{~F}$ & A & 0-Ila (LST-NG) & 10 & HGD $\ddagger$ \\
\hline & $60 \mathrm{~s}$ & M & $T$ & 0-Ila (LST-NG) & 12 & LGD§ \\
\hline & $60 \mathrm{~s}$ & $\mathrm{~F}$ & $S$ & 0-Isp & 10 & LGD \\
\hline & $50 \mathrm{~s}$ & $\mathrm{~F}$ & S & 0 -Isp & 11 & LGD \\
\hline & $60 \mathrm{~s}$ & M & A & 0-Ila (LST-NG) & 10 & HGD \\
\hline & $40 \mathrm{~s}$ & M & $S$ & 0-Ila & 8 & HGD \\
\hline \multirow{6}{*}{$\begin{array}{l}\text { 2-Examination/ } \\
\text { second F/U }\end{array}$} & $50 \mathrm{~s}$ & M & $\mathrm{T}$ & 0-Ila (LST-G) & 14 & LGD \\
\hline & $60 \mathrm{~s}$ & M & $\mathrm{T}$ & 0-Isp & 10 & LGD \\
\hline & $60 \mathrm{~s}$ & M & $\mathrm{Ra}$ & 0 -Isp & 10 & LGD \\
\hline & $50 \mathrm{~s}$ & M & $\mathrm{Rb}$ & Type 2 & 20 & Invasive caी \\
\hline & $60 \mathrm{~s}$ & $\mathrm{~F}$ & $S$ & 0-Ila (LST-NG) & 13 & HGD \\
\hline & $50 \mathrm{~s}$ & M & D & 0-Ila+IIc (LST-NG) & 12 & HGD \\
\hline \multirow{17}{*}{$\begin{array}{c}\text { 1-examination/ } \\
\text { F/U }\end{array}$} & $60 \mathrm{~s}$ & M & A & 0-IIa (LST-NG) & 16 & HGD \\
\hline & $60 \mathrm{~s}$ & M & $S$ & 0 -Is & 10 & HGD \\
\hline & $60 \mathrm{~s}$ & M & RS & $0-\mathrm{ls}$ & 9 & HGD \\
\hline & $60 s^{* *}$ & M & $T$ & 0-Ila (LST-NG) & 13 & LGD \\
\hline & $60 s^{* *}$ & $M$ & $S$ & 0-Isp & 10 & LGD \\
\hline & $50 \mathrm{~s}$ & $\mathrm{~F}$ & D & 0-Ila (LST-NG) & 10 & LGD \\
\hline & $60 \mathrm{~s}$ & M & C & 0-Ila (LST-G) & 12 & $H G D$ \\
\hline & $60 \mathrm{~s}$ & $\mathrm{~F}$ & $C$ & 0-Ila (LST-NG) & 18 & HGD \\
\hline & $60 \mathrm{~s}$ & $M$ & $\mathrm{Rb}$ & 0 -Is & 7 & HGD \\
\hline & $50 \mathrm{~s}$ & M & $S$ & 0-Ila (LST-NG) & 10 & LGD \\
\hline & $60 \mathrm{~s}$ & M & $T$ & 0-Isp & 5 & HGD \\
\hline & $60 \mathrm{~s}$ & $\mathrm{~F}$ & $\mathrm{Rb}$ & 0 -Is & 10 & LGD \\
\hline & $40 \mathrm{~s}$ & $\mathrm{~F}$ & $S$ & 0-Ila (LST-NG) & 15 & LGD \\
\hline & $60 \mathrm{~s}$ & $\mathrm{M}$ & D & 0-Ila (LST-NG) & 25 & HGD \\
\hline & $60 \mathrm{~s}$ & M & C & 0-Ila (LST-NG) & 12 & LGD \\
\hline & $60 \mathrm{~s}$ & $M$ & $T$ & 0-Ila (LST-NG) & 15 & LGD \\
\hline & $50 \mathrm{~s}$ & $M$ & A & 0-Ila (LST-NG) & 13 & LGD \\
\hline
\end{tabular}

${ }^{*} \mathrm{C}$ : caecum, A: ascending colon, T: transverse colon, D: descending colon, S: sigmoid colon, RS: rectosigmoid, Ra: upper rectum, Rb: lower rectum.

t0-Illa: flat, 0-Is: sessile, 0-Isp: semi-pedunculated, Type 2: Borrmann classification.

¥High-grade dysplasia.

§Low-grade dysplasia.

IInvasive cancer (online supplemental figure 1).

** Same patient.

HGD, high-grade dysplasia; LGD, low-grade dysplasia; LST-G, laterally spreading tumour, granular type; LST-NG, laterally spreading tumour, non-granular type. 
Table 4 Risk factors associated with metachronous advanced neoplasia

\begin{tabular}{|c|c|c|c|c|c|c|}
\hline \multirow[b]{2}{*}{ Risk factors at randomisation } & \multicolumn{2}{|c|}{ 2-examination group $(n=701)$} & \multicolumn{2}{|c|}{ 1-examination group $(n=763)$} & \multicolumn{2}{|c|}{ Both groups $(n=1464)$} \\
\hline & No $(\%)$ with $\mathrm{AN}^{*} /$ No Pts & OR $(95 \% \mathrm{Cl})$ & No (\%) with AN/No Pts & OR $(95 \% \mathrm{CI})$ & No (\%) with AN/No Pts & OR $(95 \% \mathrm{Cl})$ \\
\hline No of adenomast & & 2.25 (0.55 to 8.35$)$ & & 3.58 (1.15 to 11.11$)$ & & 2.84 (1.37 to 5.91$)$ \\
\hline $1-2$ & $6 / 343(1.7)$ & & $5 / 395(1.3)$ & & $11 / 738(1.5)$ & \\
\hline $3-4$ & $1 / 187(0.5)$ & & $3 / 197(1.5)$ & & $4 / 384(1.0)$ & \\
\hline$\geq 5$ & $5 / 171(2.9)$ & & $8 / 171(4.7)$ & & $13 / 342(3.8)$ & \\
\hline Size of largest adenomał & & $1.59(0.39$ to 5.87$)$ & & $2.25(0.72$ to 6.96$)$ & & $1.94(0.84$ to 4.40$)$ \\
\hline Small $(\leq 5 \mathrm{~mm})$ & $5 / 246(2.0)$ & & $5 / 262(1.9)$ & & $10 / 508(2.0)$ & \\
\hline Medium (6-9 mm) & $2 / 236(0.8)$ & & $3 / 263(1.1)$ & & $5 / 499(1.0)$ & \\
\hline Large $(\geq 10 \mathrm{~mm})$ & $5 / 219(2.3)$ & & $8 / 238(3.4)$ & & $13 / 457(2.8)$ & \\
\hline \multicolumn{7}{|l|}{ Morphology§ } \\
\hline Polypoid ( $\geq 10 \mathrm{~mm}$ ) & & 1.08 (0.19 to 4.38$)$ & & $1.06(0.25$ to 3.55$)$ & & 1.06 (0.38 to 2.63$)$ \\
\hline Absent & $9 / 535(1.7)$ & & $12 / 580(2.1)$ & & $21 / 1115(1.9)$ & \\
\hline Present & $3 / 166(1.8)$ & & $4 / 183(2.2)$ & & $7 / 349(2.0)$ & \\
\hline Flat $(<10 \mathrm{~mm})$ & & 0.99 (0.26 to 4.54$)$ & & - & & 3.04 (1.01 to 12.11$)$ \\
\hline Absent & $4 / 232(1.7)$ & & $0 / 255(0)$ & & $4 / 487(0.8)$ & \\
\hline Present & $8 / 469(1.7)$ & & $16 / 508(3.1)$ & & $24 / 977(2.5)$ & \\
\hline Depressed & & - & & 2.55 (0.06 to 18.34$)$ & & 1.63 (0.04 to 10.52$)$ \\
\hline Absent & $12 / 688(1.7)$ & & $15 / 743(2.0)$ & & $27 / 1431(1.9)$ & \\
\hline Present & $0 / 13(0)$ & & $1 / 20(5.0)$ & & 1/33 (3.0) & \\
\hline LST-G & & 1.60 (0.36 to 11.57$)$ & & - & & $0.77(0.02$ to 4.84$)$ \\
\hline Absent & $11 / 663(1.7)$ & & $16 / 734(2.2)$ & & 27/1397 (1.9) & \\
\hline Present & $1 / 38(2.6)$ & & $0 / 29(0)$ & & $1 / 67(1.5)$ & \\
\hline LST-NG & & 3.04 (0.07 to 22.84$)$ & & 10.16 (2.59 to 33.84$)$ & & $6.61(2.11$ to 17.61$)$ \\
\hline Absent & $11 / 680(1.6)$ & & $11 / 726(1.5)$ & & $22 / 1406(1.6)$ & \\
\hline Present & $1 / 21(4.8)$ & & $5 / 37(13.5)$ & & $6 / 58(10.3)$ & \\
\hline $\begin{array}{l}\text { Pathologically advanced } \\
\text { adenomas }\end{array}$ & & $1.20(0.30$ to 4.45$)$ & & 2.81 (0.91 to 9.50$)$ & & $1.92(0.92$ to 4.00$)$ \\
\hline Absent & $7 / 439(1.6)$ & & $6 / 475(1.3)$ & & $13 / 914(1.4)$ & \\
\hline Present & $5 / 262(1.9)$ & & $10 / 288(3.5)$ & & $15 / 550(2.7)$ & \\
\hline $\begin{array}{l}\text { Large serrated lesion }(\geq 10 \mathrm{~mm}, \\
\text { Rt-sided }^{* *} \text { ) }\end{array}$ & & - & & - & & - \\
\hline Absent & $12 / 693(1.7)$ & & $16 / 755(2.1)$ & & $28 / 1448(1.9)$ & \\
\hline Present & $0 / 8(0)$ & & $0 / 8(0)$ & & $0 / 16(0)$ & \\
\hline Age & & $1.18(0.32$ to 4.76$)$ & & 2.88 (0.86 to 12.36$)$ & & $1.88(0.86$ to 4.13$)$ \\
\hline$<60$ years & $5 / 320(1.6)$ & & $4 / 370(1.1)$ & & $9 / 690(1.3)$ & \\
\hline$\geq 60$ years & $7 / 381(1.8)$ & & 12/393 (3.1) & & $19 / 774(2.5)$ & \\
\hline Gender & & 1.01 (0.27 to 4.63$)$ & & 1.67 (0.50 to 7.16$)$ & & $1.32(0.56$ to 3.00$)$ \\
\hline Female & $4 / 235(1.7)$ & & $4 / 271(1.5)$ & & 8/506 (1.6) & \\
\hline Male & $8 / 466(1.7)$ & & $12 / 492(2.4)$ & & $20 / 958(2.1)$ & \\
\hline Family history of $\mathrm{CRC}+\dagger$ & & $2.78(0.60$ to 10.59$)$ & & $2.23(0.51$ to 7.55$)$ & & $2.39(1.07$ to 5.35$)$ \\
\hline Absent & $8 / 592(1.4)$ & & $12 / 662(1.8)$ & & $20 / 1254(1.6)$ & \\
\hline Present & $4 / 109(3.7)$ & & $4 / 101(4.0)$ & & $8 / 210(3.8)$ & \\
\hline
\end{tabular}

*Any lesion with low-grade dysplasia $\geq 10 \mathrm{~mm}$, high-grade dysplasia.

TOR; number of adenomas ( $\geq 5$ vs $\leq 4$ ).

$\ddagger 0 R$; size of largest adenoma ( $\geq 10 \mathrm{~mm}$ vs $\leq 9 \mathrm{~mm}$ ).

§Polypoid; 0-Ip, 0-Isp, 0-Is type, Flat; 0-Ila type, Depressed; 0-Ila+IIc, 0-IIc type.

ПAny low-grade dysplasia $\geq 10 \mathrm{~mm}$, high-grade dysplasia.

${ }^{* *}$ Caecum, ascending colon, transverse colon.

††First-degree relatives.

CRC, colorectal cancer; LST-G, laterally spreading tumour, granular type; LST-NG, laterally spreading tumour, non-granular type.

not necessary to perform intensive surveillance colonoscopies at 1 and 3 years, and a surveillance colonoscopy at 3 years after polypectomy is sufficient. Our study confirmed the finding originally reported by the NPS over 20 years ago, but currently with a focus on NP-CRNs. Despite the similarity between our study and that of the NPS, we are convinced that our study provides new important knowledge associated with the following strengths.

First, the fact that a two-round colonoscopy was performed at baseline is a unique strength of this study. It enabled more accurate detection and complete removal of neoplastic lesions during the two-round baseline colonoscopy before randomisation. Hence, findings obtained from this study were based on a valid assumption that all neoplastic lesions were removed at baseline colonoscopy. The high quality of colonoscopies that were performed with high-definition video endoscopes under good bowel preparation in this study further strengthens this point. In this context, the low incidence of $\mathrm{AN}(1.9 \%, \mathrm{n}=28)$ and invasive cancer $(0.07 \%, \mathrm{n}=1)$ observed in this study is comprehensible; the incidence of AN here is lower than that in other studies, including the NPS (3.3\%). ${ }^{7-10}$ However, it is noteworthy that 
AN were still observed in $1.9 \%$ of patients, even after a tworound high-quality colonoscopy. The evaluation of the characteristics of the AN detected in this condition must be informative to investigate the cause of PCCRC.

From this perspective, the detailed characterisation of detected AN with a special focus on NP-CRNs is another notable strength of this study. Due to the high-quality colonoscopies performed by experienced endoscopists, which enabled the accurate detection of flat lesions, this study clearly showed that over $60 \%$ of detected AN were morphologically NP-CRNs, and over $80 \%$ of them were classified as LST-NG lesions. ${ }^{29-31}$ Considering such a high proportion of LST-NG among detected AN and the wellknown fact that LST-NG has an easily-overlooked appearance and different oncogenic mutations in KRAS, BRAF or PIK3CA from polypoid lesions, ${ }^{34-36}$ it can be postulated that LST-NG may be an important precursor of PCCRC. The relatively high proportion of LST-NG detected in the right-sided colon in this study is also in agreement with the fact that many PCCRCs are located in the right-sided colon. ${ }^{37-40}$ Although further studies are required to confirm the relationship between LST-NG and PCCRC, this study is supposedly provides sufficient information suggesting the potential significance of LST-NG as a precursor of PCCRC. The clinical significance of LST-NG is also supported by the important finding obtained from this study that the presence of LST-NG lesions at the baseline colonoscopies is a strong risk factor for AN detected at follow-up colonoscopy. In addition to the potential nature of LST-NG as a precursor of PCCRC, its potential association with PCCRC at a different site is worth further investigation.

The possible causes of AN detected at surveillance in this study included lesions missed at baseline colonoscopy and newly developed lesions (no AN was diagnosed as a residual/ recurrent lesion after endoscopic treatment). Because of the relatively short follow-up period, oversights at baseline colonoscopy cannot be denied even with a two-round high-quality colonoscopy. This emphasises the importance of the effort to detect all clinically important lesions, including LST-NG, during colonoscopy. Furthermore, considering that the AN detected during surveillance in this study mainly comprised LST-NG, careful endoscopic observation with sufficient attention to LST-NG is also recommended in surveillance colonoscopy, particularly for high-risk individuals with a history of being treated for LST-NG. In addition to the effort by endoscopists, further development of endoscopic technologies such as image-enhanced endoscopy and artificial intelligence is warranted. ${ }^{41-45}$

Although the non-inferiority of the 3-year surveillance colonoscopy compared with 1-year and 3-year surveillance colonoscopies in terms of effectively detecting AN was confirmed in this study, it does not indicate that every patient should uniformly undergo surveillance colonoscopy 3 years after polypectomy. On the basis of the extremely low incidence of AN observed in this study, it may be possible to prolong the surveillance intervals for patients with a low risk of incidence of metachronous AN. As believed, it is essential to establish surveillance programmes after considering AN risks (that varies with patients) as recommended by the recent postpolypectomy surveillance guidelines. ${ }^{11-13}$ In this study, as shown in table 4 , the presence of $\geq 5$ adenomas, flat lesions sized $<10 \mathrm{~mm}$, and LST-NG at baseline colonoscopy and the presence of CRC family history were identified as risk factors for the incidence of AN (although, if analysed separately for the two group arms, some of the factors were not identified as significant risk factors presumably due to the small number of cases). The significance of the presence of $\geq 5$ adenomas as a risk factor for metachronous $\mathrm{AN}$ is compatible with the descriptions and recommendations of the recent guidelines ${ }^{11-13}$; however, other factors including the presence of LST-NG which was the strongest risk factor in this study were not considered in the current surveillance guidelines. A prolonged surveillance interval ( $>3$ years) may be proposed for patients without the above-mentioned risk factors, as recent guidelines recommend longer surveillance intervals for patients with $<5$ adenomas at baseline colonoscopy. On the other hand, patients with these risk factors need to be considered at high risk in surveillance. Particularly those with LST-NG at baseline, who were shown to have the highest risk for the incidence of metachronous $\mathrm{AN}$, require extremely careful attention. The $10.3 \%$ incidence of AN observed during surveillance in patients with LST-NG at baseline in this study indicates the necessity of surveillance colonoscopies at intervals that are no longer than 3 years for these patients. Based on the finding that 2-examination group showed a remarkably lower AN incidence (4.8\%) than 1-examination group (13.5\%) among those with LST-NG at baseline, a surveillance interval shorter than 3 years as set in 2-examination group may be worth considering only for these high-risk individuals. Even if this short surveillance interval is employed for those individuals, it would have minimal influence on the colonoscopy capacity because of the low prevalence of LST-NG in the screening population. The necessity of considering the presence of LST-NG at baseline as an important factor to classify patients as being at high risk and requiring more frequent surveillance is a novel and essential finding that can contribute to our existing knowledge on this subject. ${ }^{11-13}$ The significance of the presence of small-sized $(<10 \mathrm{~mm})$ flat lesions in terms of AN risk was also shown in this study. These small flat lesions included precursors for LST-NG, and the significance is believed to strengthen the importance of LST-NG. The influence of CRC family history, as observed in this study ${ }_{2}$ is also notable and is compatible with the findings of a recent study, ${ }^{46}$ and further evaluation is warranted. Currently, we continue to follow up the long-term clinical outcomes, including CRC incidence and mortality, of this study cohort (JPS cohort), ${ }^{23}$ and the long-term follow-up data are expected to provide informative knowledge regarding the optimal risk-stratified surveillance intervals.

This study had several limitations. First, high expertise in all participating institutions may be a limitation in terms of generalisation of the obtained findings from this study. However, considering this study's focus on NP-CRNs, we believe that the study was conducted in the ideal setting in which high-quality colonoscopy could accurately detect NP-CRNs. Second, the study design, involving a single country (Japan), also limits the generalisability of the results. Considering the potential difference in the prevalence of colorectal lesions, including LST-NG, further studies, assessing high-quality colonoscopies in other regions, are warranted. Third, the information of the allocation status on the two groups (1-examination group or 2-examination group) were not blinded to the participating endoscopists. Although the nonadvanced adenomas, mainly composed of diminutive adenomas $(\leq 5 \mathrm{~mm})$, were more frequently detected in the 2-examination group, it is not believed to be related to this limitation. Presumably, the more frequent detection of diminutive polyps in the 2-examination group was caused by exposure to more frequent colonoscopies. With the increased use of colonoscopies, the number of detected diminutive polyps has reportedly increased, and how to manage the diminutive polyps is another important 
topic to address. ${ }^{47} 48$ Fourth, serrated lesions were not fully examined in this study because the evaluation of serrated lesions was not standardised, as presented during the study period. However, pathological evaluation of resected lesions was carefully performed by specialists. In particular, all detected AN were evaluated through a central pathological judgement by selected board-certified pathologists with expertise in gastrointestinal pathology. Further, qualitative diagnoses, considering serrated lesions, including the differential diagnosis between LST-NG and serrated lesions, was appropriately performed. Finally, in the assessment of risk factors for metachronous AN, several patient factors, including lifestyle factors, that were potentially associated with colorectal neoplasia were not fully examined. ${ }^{49} 50$ Thus, the significance of CRC family history as a risk factor for AN requires further evaluation with adjustments for more potential confounding factors.

In conclusion, a single colonoscopy at 3 years after a tworound baseline colonoscopy detected AN with equal accuracy compared with follow-up colonoscopies performed at 1 and 3 years postpolypectomy, even considering NP-CRNs. The fact that even after a two-round baseline colonoscopy, AN mainly composed of LST-NG that were detected within 3 years, emphasises the importance of paying careful attention to these lesions.

\author{
Author affiliations \\ ${ }^{1}$ Cancer Screening Center, National Cancer Center Hospital, Tokyo, Japan \\ ${ }^{2}$ Endoscopy Division, National Cancer Center Hospital, Tokyo, Japan \\ ${ }^{3}$ TF Clinic, Tokyo, Japan \\ ${ }^{4}$ Gastrointestinal Center and Institute of Minimally Invasive Endoscopic Care (iMEC), \\ Sano Hospital, Kobe, Hyogo, Japan \\ ${ }^{5}$ Digestive Disease Center, Showa University Northern Yokohama Hospital, Yokohama, \\ Kanagawa, Japan \\ ${ }^{6} \mathrm{Oda} \mathrm{GI}$ Endoscopy and Gastroenterology Clinic, Kumamoto, Japan \\ 'Division of Endoscopy, Shizuoka Cancer Center, Sunto-gun, Shizuoka, Japan \\ ${ }^{8}$ Division of Pathology, Shizuoka Cancer Center, Sunto-gun, Shizuoka, Japan \\ ${ }^{9}$ Department of Gastroenterology, Tochigi Cancer Center, Utsunomiya, Tochigi, Japan \\ ${ }^{10}$ Division of Gastroenterology, Department of Medicine, Showa University School of \\ Medicine, Tokyo, Japan \\ ${ }^{11}$ Department of Gastroenterology and Endoscopy, National Cancer Center Hospital \\ East, Kashiwa, Chiba, Japan \\ ${ }^{12}$ Department of Gastrointestinal Oncology, Osaka International Cancer Institute, \\ Osaka, Japan \\ ${ }^{13}$ Department of Gastroenterology, The Cancer Institute Hospital of Japanese \\ Foundation for Cancer Research, Tokyo, Japan \\ ${ }^{14}$ Department of Gastroenterology, Kitasato University East Hospital, Sagamihara, \\ Kanagawa, Japan \\ ${ }^{15}$ Hattori GI Endoscopy and Gastroenterology Clinic, Kumamoto, Japan \\ ${ }^{16}$ Department of Gastroenterology, Saku Central Hospital Advanced Care Center, \\ Saku, Nagano, Japan \\ ${ }^{17}$ Department of Molecular-Targeting Cancer Prevention, Graduate School of Medical \\ Science, Kyoto Prefectural University of Medicine, Kyoto, Japan \\ ${ }^{18}$ Department of Medical Statistics, Toho University, Tokyo, Japan \\ ${ }^{19}$ Pathology and Clinical Laboratory Division, JR Tokyo General Hospital, Tokyo, Japan \\ ${ }^{20}$ Department of Pathology, Shinko Hospital, Kobe, Hyogo, Japan \\ ${ }^{21}$ Division of Molecular and Diagnostic Pathology, Niigata University Graduate School \\ of Medical and Dental Sciences, Niigata, Japan \\ ${ }^{22}$ Aomori Prefectural Central Hospital, Aomori, Japan
}

Acknowledgements We would like to thank all investigators who cooperated in recruiting and following up patients at the 11 participating centres, data management staff of the Medical Research Support (Tomoko Saeki, Kumi Okazaki, Naoko Sawada, Natsuko Nishida), monitoring committee members (Tetsu Shinkai (Chair),Hiroyasu Esumi, Atsushi Ochiai, Yasuhiro Shimada, Takayuki Akasu), Hiroshi Saito and Kuang-I Fu, for giving us valuable advice regarding the organisation of the Japan Polyp Study Workgroup and study protocol.

Contributors Conception and design: TM, TFujii, YSan, S-eK, YO, KHo, TShim, YSai, NK, MI, HIs, YM, and SY, Data collection: TM, TFujii, YSan, S-eK, YO, KHo, YSai, NK, MSe, KKon, HIK, Hli, YT, MI, KKob, MSa, YY, KHa, and TShin, Analysis and interpretation of the data: HIs and YM, Pathological central review: TShim, HT, TFujim, and YA, Drafting the article: TM, MSe, Critical revision of the article for important intellectual content: TFujii, YSan, S-eK, YO, KHo, TShim, YSai, NK, MSe, HIs, YM, and
SY, Final approval of the article: TM, TFujii, YSan, S-eK, YO, KHo, TShim, YSai, NK, MSe, KKon, HIlK, Hli, YT, MI, KKob, MSa, YY, KHa, TShin, HIs, YM, HT, TFujim, YA and SY.

Funding This study was supported by the Grants-in-Aid for Clinical Cancer Research (13S-8, 16S-33 and 20S-12) from the Ministry of Health, Labour and Welfare, Japan, the National Cancer Center Research and Development Fund (29-A13, 30-A-16) and the Japan Agency for Medical Research and Development (AMED; JP16ck0106102, JP19ck0106276).

Competing interests None declared.

Patient and public involvement Patients and/or the public were not involved in the design, or conduct, or reporting, or dissemination plans of this research.

Patient consent for publication Not required.

Ethics approval The study protocol was approved by the Institutional Review Board of each participating hospital. This study was registered with the University Hospital Medical Information Network Clinical Trials Registry (UMIN-CTR number, UMIN C000000058).

Provenance and peer review Not commissioned; externally peer reviewed.

Data availability statement Data are available on reasonable request. All analyses relevant to the study are included in the article. All data requests should be submitted to the corresponding author for consideration. Access to anonymised data may be granted following review.

Open access This is an open access article distributed in accordance with the Creative Commons Attribution Non Commercial (CC BY-NC 4.0) license, which permits others to distribute, remix, adapt, build upon this work non-commercially, and license their derivative works on different terms, provided the original work is properly cited, appropriate credit is given, any changes made indicated, and the use is non-commercial. See: http://creativecommons.org/licenses/by-nc/4.0/.

\section{ORCID iDs}

Takahisa Matsuda http://orcid.org/0000-0002-9244-2820

Takahiro Fujii http://orcid.org/0000-0001-8190-8907

Yasushi Sano http://orcid.org/0000-0002-3352-5757

Shin-ei Kudo http://orcid.org/0000-0002-4268-1217

Kinichi Hotta http://orcid.org/0000-0001-5608-0968

Yutaka Saito http://orcid.org/0000-0003-2296-8373

Masau Sekiguchi http://orcid.org/0000-0001-6253-6896

Kazuo Konishi http://orcid.org/0000-0002-2390-274X

Hiroaki Ikematsu http://orcid.org/0000-0001-9840-4588

Hiroyasu lishi http://orcid.org/0000-0002-8850-0274

Yoji Takeuchi http://orcid.org/0000-0003-3814-298X

Masahiro Igarashi http://orcid.org/0000-0002-1392-0389

Kiyonori Kobayashi http://orcid.org/0000-0003-4399-0940

Miwa Sada http://orcid.org/0000-0003-3057-8762

Tomoaki Shinohara http://orcid.org/0000-0003-4592-9895

Hideki Ishikawa http://orcid.org/0000-0001-5980-698X

Yoshitaka Murakami http://orcid.org/0000-0002-8134-0327

Hirokazu Taniguchi http://orcid.org/0000-0003-4212-4776

Yoichi Ajioka http://orcid.org/0000-0002-7532-5454

\section{REFERENCES}

1 Bray F, Ferlay J, Soerjomataram I, et al. Global cancer statistics 2018: GLOBOCAN estimates of incidence and mortality worldwide for 36 cancers in 185 countries. CA Cancer J Clin 2018;68:394-424.

2 Winawer SJ, Zauber AG, Ho MN, et al. Prevention of colorectal cancer by colonoscopic polypectomy. The National Polyp study Workgroup. N Engl J Med 1993;329:1977-81.

3 Brenner $\mathrm{H}$, Chang-Claude J, Seiler CM, et al. Protection from colorectal cancer after colonoscopy: a population-based, case-control study. Ann Intern Med 2011;154:22-30

4 Zauber AG, Winawer SJ, O'Brien MJ, et al. Colonoscopic polypectomy and long-term prevention of colorectal-cancer deaths. N Engl J Med 2012;366:687-96.

5 Baxter NN, Warren JL, Barrett MJ, et al. Association between colonoscopy and colorectal cancer mortality in a US cohort according to site of cancer and colonoscopist specialty. J Clin Oncol 2012;30:2664-9.

6 Nishihara R, Wu K, Lochhead P, et al. Long-Term colorectal-cancer incidence and mortality after lower endoscopy. N Engl J Med 2013;369:1095-105.

7 Winawer SJ, Zauber AG, O'Brien MJ, et al. Randomized comparison of surveillance intervals after colonoscopic removal of newly diagnosed adenomatous polyps. The National Polyp study Workgroup. N Engl J Med 1993;328:901-6.

8 van Stolk RU, Beck GJ, Baron JA, et al. Adenoma characteristics at first colonoscopy as predictors of adenoma recurrence and characteristics at follow-up. The polyp prevention Study Group. Gastroenterology 1998;115:13-18.

9 Martínez ME, Sampliner R, Marshall JR, et al. Adenoma characteristics as risk factors for recurrence of advanced adenomas. Gastroenterology 2001;120:1077-83.

10 Lieberman DA, Weiss DG, Harford WV, et al. Five-Year colon surveillance after screening colonoscopy. Gastroenterology 2007;133:1077-85. 
11 Gupta S, Lieberman D, Anderson JC, et al. Recommendations for follow-up after colonoscopy and polypectomy: a consensus update by the US Multi-Society Task force on colorectal cancer. Gastroenterology 2020;158:1131-53.

12 Hassan C, Antonelli G, Dumonceau J-M, et al. Post-polypectomy colonoscopy surveillance: European Society of Gastrointestinal Endoscopy (ESGE) Guideline Update 2020. Endoscopy 2020;52:687-700.

13 Rutter MD, East J, Rees CJ, et al. British Society of Gastroenterology/Association of Coloproctology of great britain and Ireland/Public health England postpolypectomy and post-colorectal cancer resection surveillance guidelines. Gut 2020;69:201-23.

14 East JE, Atkin WS, Bateman AC, et al. British Society of gastroenterology position statement on serrated polyps in the colon and rectum. Gut 2017:66:1181-96.

15 Baxter NN, Sutradhar R, Forbes SS, et al. Analysis of administrative data finds endoscopist quality measures associated with postcolonoscopy colorectal cancer. Gastroenterology 2011;140:65-72.

16 Cooper GS, Xu F, Barnholtz Sloan JS, et al. Prevalence and predictors of interval colorectal cancers in Medicare beneficiaries. Cancer 2012;118:3044-52.

17 Singh S, Singh PP, Murad MH, et al. Prevalence, risk factors, and outcomes of interval colorectal cancers: a systematic review and meta-analysis. Am J Gastroenterol 2014;109:1375-89.

18 Rutter MD, Beintaris I, Valori R, et al. World endoscopy organization consensus statements on post-colonoscopy and post-imaging colorectal cancer. Gastroenterology 2018;155:909-25.

19 Fujii T, Rembacken BJ, Dixon MF, et al. Flat adenomas in the United Kingdom: are treatable cancers being missed? Endoscopy 1998;30:437-43.

20 Kudo S, Kashida H, Tamura T, et al. Colonoscopic diagnosis and management of nonpolypoid early colorectal cancer. World I Surg 2000;24:1081-90.

21 Rembacken BJ, Fujii T, Cairns A, et al. Flat and depressed colonic neoplasms: a prospective study of 1000 colonoscopies in the UK. Lancet 2000;355:1211-4.

22 Soetikno RM, Kaltenbach T, Rouse RV, et al. Prevalence of nonpolypoid (flat and depressed) colorectal neoplasms in asymptomatic and symptomatic adults. JAMA 2008;299:1027-35.

23 Sano Y, Fujii T, Matsuda T, et al. Study design and patient recruitment for the Japan polyp study. Open Access J of Clin Trials 2014;6:37-44.

24 Matsuda T, Fujii T, Sano Y, et al. Five-Year incidence of advanced neoplasia after initia colonoscopy in Japan: a multicenter retrospective cohort study. Jpn J Clin Oncol 2009:39:435-42.

25 Foss FA, Milkins S, McGregor AH. Inter-Observer variability in the histological assessment of colorectal polyps detected through the NHS bowel cancer screening programme. Histopathology 2012;61:47-52.

26 Mahajan D, Downs-Kelly E, Liu X, et al. Reproducibility of the villous component and high-grade dysplasia in colorectal adenomas $<1 \mathrm{~cm}$ : implications for endoscopic surveillance. Am I Surg Pathol 2013;37:427-33.

27 ASGE Standards of Practice Committee, Saltzman JR, Cash BD, et al. Bowel preparation before colonoscopy. Gastrointest Endosc 2015;81:781-94.

28 Participants in the Paris Workshop. The Paris endoscopic classification of superficial neoplastic lesions: esophagus, stomach, and colon: November 30 to December 1 , 2002. Gastrointest Endosc 2003;58:S3-43.

29 Saito $\mathrm{Y}$, Fujii T, Kondo H, et al. Endoscopic treatment for laterally spreading tumors in the colon. Endoscopy 2001;33:682-6.

30 Uraoka T, Saito Y, Matsuda T, et al. Endoscopic indications for endoscopic mucosal resection of laterally spreading tumours in the colorectum. Gut 2006:55:1592-7.

31 Sano Y, Chiu H-M, Li X-B, et al. Standards of diagnostic colonoscopy for early-stage neoplasia: recommendations by an Asian private group. Dig Endosc 2019:31:227-44.
32 Hamilton SR, Aaltonen LA. World Health organization classification of tumours: pathology and genetics of tumours of the digestive system. Lyon, France: IARC Press, 2000: 104-19

33 Japanese Society for Cancer of the Colon and Rectum. General rules for clinical and pathological studies on cancer of the colon, rectum and anus. 6th edn. Tokyo: Kanehara \& CO., LTD, 1998.

34 Hiraoka S, Kato J, Tatsukawa M, et al. Laterally spreading type of colorectal adenoma exhibits a unique methylation phenotype and K-ras mutations. Gastroenterology 2006;131:379-89.

35 Sanduleanu S, Masclee AM, Meijer GA. Interval cancers after colonoscopy-insights and recommendations. Nat Rev Gastroenterol Hepatol 2012;9:550-4.

36 Chang L-C, Chiu H-M, Shun C-T, et al. Mutational profiles of different macroscopic subtypes of colorectal adenoma reveal distinct pathogenetic roles for KRAS, BRAF and PIK3CA. BMC Gastroenterol 2014;14:221.

37 Bressler B, Paszat LF, Vinden C, et al. Colonoscopic miss rates for right-sided colon cancer: a population-based analysis. Gastroenterology 2004;127:452-6.

38 Singh H, Nugent Z, Demers AA, et al. The reduction in colorectal cancer mortality after colonoscopy varies by site of the cancer. Gastroenterology 2010;139:1128-37.

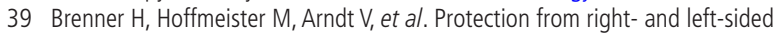
colorectal neoplasms after colonoscopy: population-based study. J Natl Cancer Inst 2010;102:89-95.

40 Sanduleanu S, le Clercq CM, Dekker E, et al. Expert Working Group on 'Rightsided lesions and interval cancers', Colorectal Cancer Screening Committee, World Endoscopy Organization. Definition and taxonomy of interval colorectal cancers: a proposal for standardising nomenclature. Gut 2015;64:1257-67.

41 Matsuda T, Ono A, Sekiguchi M, et al. Advances in image enhancement in colonoscopy for detection of adenomas. Nat Rev Gastroenterol Hepatol 2017;14:305-14.

42 Ikematsu H, Sakamoto T, Togashi K, et al. Detectability of colorectal neoplastic lesions using a novel endoscopic system with blue laser imaging: a multicenter randomized controlled trial. Gastrointest Endosc 2017;86:386-94.

43 Mori Y, Kudo S-E, Misawa M, et al. Real-time use of artificial intelligence in identification of diminutive polyps during colonoscopy: a prospective study. Ann Intern Med 2018;169:357-66.

44 Takeuchi Y, Sawaya M, Oka S, et al. Efficacy of autofluorescence imaging for flat neoplasm detection: a multicenter randomized controlled trial (A-FLAT trial). Gastrointest Endosc 2019;89:460-9.

45 Atkinson NSS, Ket S, Bassett P, et al. Narrow-band imaging for detection of neoplasia at colonoscopy: a meta-analysis of data from individual patients in randomized controlled trials. Gastroenterology 2019;157:462-71.

46 Jacobs ET, Gupta S, Baron JA, et al. Family history of colorectal cancer in firstdegree relatives and metachronous colorectal adenoma. Am I Gastroenterol 2018;113:899-905.

47 Brenner H, Altenhofen L, Kretschmann J, et al. Trends in adenoma detection rates during the first 10 years of the German screening colonoscopy program. Gastroenterology 2015;149:356-66.

48 Sekiguchi M, Otake Y, Kakugawa Y, et al. Incidence of advanced colorectal neoplasia in individuals with untreated diminutive colorectal adenomas diagnosed by magnifying image-enhanced endoscopy. Am J Gastroenterol 2019;114:964-73.

49 Yeoh K-G, Ho K-Y, Chiu H-M, et al. The Asia-Pacific colorectal screening score: a validated tool that stratifies risk for colorectal advanced neoplasia in asymptomatic Asian subjects. Gut 2011:60:1236-41.

50 Sekiguchi M, Kakugawa Y, Matsumoto M, et al. A scoring model for predicting advanced colorectal neoplasia in a screened population of asymptomatic Japanese individuals. J Gastroenterol 2018:53:1109-19. 